\section{Frederico Simões Barbosa, um pesquisador à frente do seu tempo}

\author{
Frederico Simões Barbosa: a researcher ahead of \\ his time
}

\author{
Frederico Simões Barbosa: un investigador \\ adelantado a su tiempo
} adelantado a su tiempo 
Reunião dos pesquisadores em esquistossomose da Fundação Oswaldo Cruz. Petrópolis, 1988 (Acervo particular do autor). Da esquerda para a direita: Andre Furtado, Naftale Katz, Frederico Simões Barbosa, Amaury Coutinho, Luis Rey, Sergio Arouca, Carlos Morel e Fernando Avila Pires.

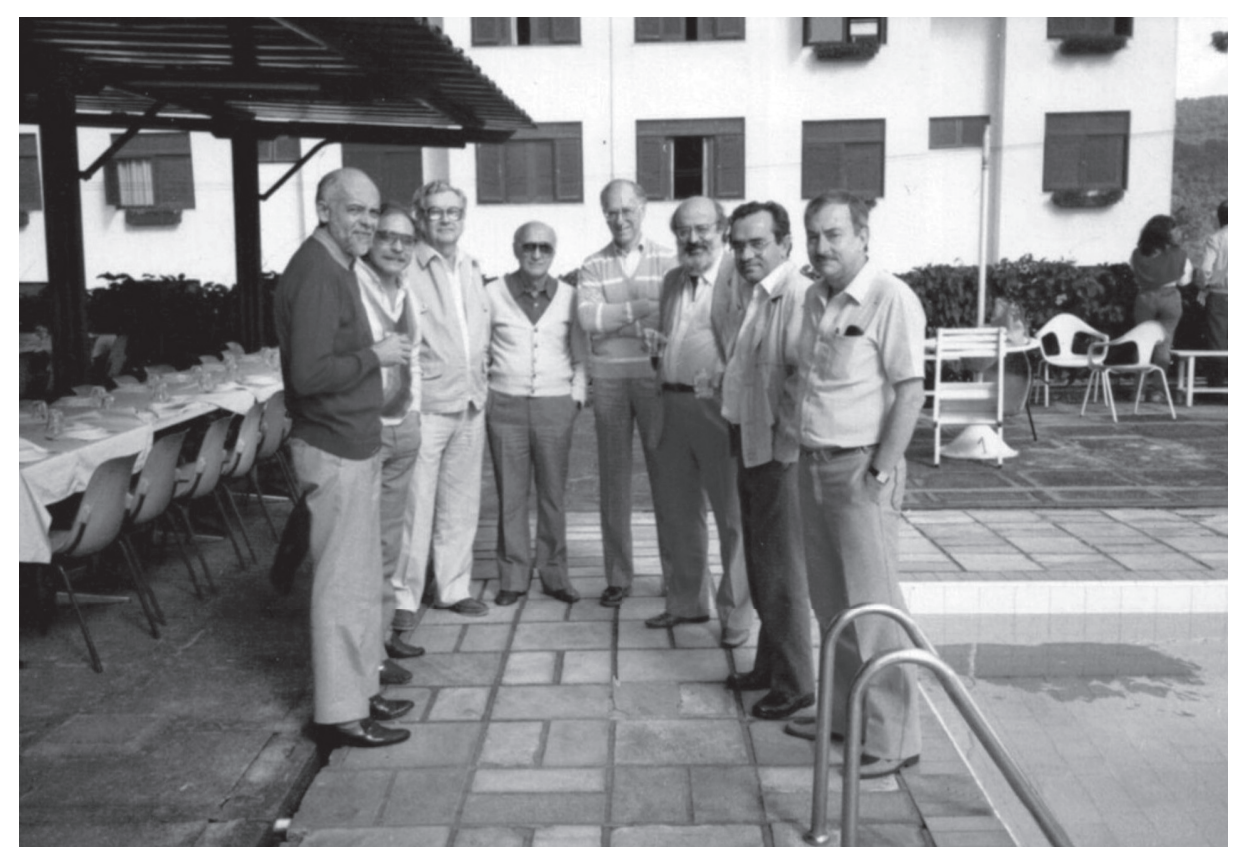

cussões, às vezes um pouco exacerbadas com Wladimir Lobato Paraense, outro malacologista brasileiro de renome internacional. Em vários congressos científicos a apresentação dos temas pelos dois ilustres pesquisadores era aguardada com muita ansiedade pelos especialistas, seja pelas informações que traziam, seja pela polêmica que não negavam em fazer em público, sempre com intenso brilhantismo.

Barbosa prosseguiu seus estudos em esquistossomose nas áreas da morbidade 18 e epidemiologia. Fez investigações epidemiológicas em várias comunidades do Nordeste 19,20,21 e correlação entre a prevalência, morbidade e "qualidade dos vetores". Conceituou o termo qualidade de vetor, ou seja, "as condições que fazem um transmissor eficiente ou não".

Em 1968, a Escola de Engenharia da Universidade Federal de Minas Gerais, em Belo Horizonte, junto com a Organização Pan-Americana da Saúde (OPAS), promoveram um curso de especialização em saneamento ambiental. Foi durante esse curso que eu, como aluno e professor do mesmo, tive o prazer e a honra de estreitar as relações com o Frederico Simões Barbosa. Posso dizer que seus trabalhos foram sempre inspiração para os meus.

Um trabalho publicado por Fred em 1971 (mas realizado de 1960 a 1968) trouxe uma grande contribuição ao controle dessa endemia, quando em Pontezinha, Pernambuco, ele conseguiu o controle da esquistossomose por interrupção da transmissão com a utilização do saneamento ambiental e desenvolvimento comunitário ${ }^{22}$. Essa preciosa informação só teve a sua importância reconhecida pela comunidade científica e pelos serviços de saúde muitos anos depois.

No início da década de 1950, foi nomeado primeiro Diretor do Centro de Pesquisas Aggeu Magalhães (CPqAM), subordinado à Divisão da Organização Sanitária, que após alguns anos passou a pertencer ao Instituto Nacional de Endemias Rurais (INERu). O INERu que foi criado para desenvolver pesquisas operacionais visando ao controle das principais endemias parasitárias brasileiras teve mais três centros adicionados, a saber: o de Belo Horizonte, Rio de Janeiro e Salvador. Fred estruturou o CPqAM para realizar pesquisas e em pouco tempo o Centro passou a ser reconhecido nacional e internacionalmente. 
Permaneceu no CPqAM de 1950 a 1962 e de 1964 a 19689.

Foi sócio-fundador da Sociedade Brasileira de Medicina Tropical (SBMT) e seu Presidente de 1977 a 1979. Na abertura do Congresso anual da SBMT fez um discurso inesquecível no qual demonstrou a origem pejorativa do nome "medicina tropical”, criada pelos ingleses para marcar negativamente a parte sul do planeta. Defendeu com razão que as doenças chamadas tropicais não existiam por determinação geográfica, mas devido às condições socioeconômicas e políticas que mantinham a maioria das populações na miséria ou pobreza, no que na época eram denominados como países subdesenvolvidos do Terceiro Mundo.

Barbosa ficou na Universidade de Brasília (UnB) de 1972 a 1981 e, sem interromper totalmente suas pesquisas sobre esquistossomose, iniciou outras atividades na área da pedagogia do ensino nas ciências da saúde, criando um "Programa Integrado da Saúde da Comunidade”, cuja principal contribuição foi mostrar que a "Universidade brasileira pode alcançar níveis de articulação ensino/serviços capazes de induzir modificações na estrutura e administração docentes, que possam levar as mudanças desejadas no processo de abertura democrática do sistema docente" 9.

Foi também na UnB que Fred teve o seu maior dissabor. O Reitor da Universidade, na época, que era um Capitão de Fragata da Marinha, indicado pelo Governo Militar (instalado em 1964), mandou abrir um inquérito contra ele, “pois havia desaparecido uma pá e algumas outras ferramentas, adquiridas com verbas de auxílio de projeto financiado pelo CNPq" (Barbosa FS, comunicação pessoal). Barbosa acreditava que havia sido o Professor Aluízio Prata o autor da denúncia e rompeu totalmente sua relação com o mesmo. Sabendo eu desse fato, e com a autorização do Fred, procurei o Professor Prata, pois conhecia pelo menos duas atividades do mesmo em defesa de pesquisadores de esquerda e que, portanto, o Fred deveria estar enganado. Após algumas idas e vindas, foi possível fazer o encontro dos mesmos em que tudo ficou esclarecido, e o Fred convencido da total inocência do Prata. Essa acusação non sense era comum durante o período da ditadura militar no meio científico e em outros ambientes.

Indo para a Escola Nacional de Saúde Pública da Fundação Oswaldo Cruz, Fred continuou sempre trabalhando e desenvolvendo estudos de epidemiologia social. Suas contribuições nesse período serão relatadas por aqueles que conviveram com ele.

Publicou quase duas centenas de trabalhos nos seus 88 anos de vida.

Excelente professor, trabalhador de campo, administrador, investigador de alta sensibilidade e rígida metodologia científica, associou as suas pesquisas à busca de soluções de problemas brasileiros, sempre com vistas ao bem-estar das comunidades mais carentes, as quais sempre respeitou e dedicou sua vida de trabalho.
1. Coutinho EM. Frederico Adolfo Simões Barbosa (1916-2004). Rev Soc Bras Med Trop 2004; 37:427-8.

2. Barbosa FS, Dubbin JE. Estrutura interna dos Australorbis (Mollusca, Planorbidae) de Pernambuco e outros estados. Publicações Avulsas do Instituto Aggeu Magalhães 1951; 1:1-6.

3. Barbosa FS, Silva GM. Curvas de crescimento de Australorbis glabratus e sua aplicação à epidemiologia e à profilaxia da esquistossomose. Publicações Avulsas do Instituto Aggeu Magalhães 1951; 1:35-42.

4. Barbosa FS, Dobbin JE, Vieira AE. Inquérito preliminar sobre infestação de planorbídeos de alguns municípios de Pernambuco. Publicações Avulsas do Instituto Aggeu Magalhães 1952; 1:99-124.
5. Barbosa FS, Dobbin JE, Coelho MV. Infestação natural de Rattus rattus frugivorus por Schistosoma mansoni em Pernambuco. Publicações Avulsas do Instituto Aggeu Magalhães 1953; 2:43-6.

6. Barbosa FS, Coelho MV. Infestação natural do $D i$ delphis paraguayensis paraguayensis (Marsupialia, Didelphidae) por Schistosoma mansoni em Pernambuco. Publicações Avulsas do Instituto Aggeu Magalhães 1954; 3:1-4.

7. Barbosa FS, Calado OB, Moraes JG, Almeida AM. Ação moluscocida sinérgica da saponina de Sapidus saponaria e pentaclorofenato de sódio. Publicações Avulsas do Instituto Aggeu Magalhães 1952; $1: 129-40$. 
8. Dobrovolny CG, Barbosa FS. Field trials of sodium pentachlorophenate as a molluscicide in flowing waters in Brasil. Publicações Avulsas do Instituto Aggeu Magalhães 1953; 2:121-58.

9. Barbosa FS. Memorial apresentado à Escola Nacional de Saúde Pública, Fundação Oswaldo Cruz, para concorrer ao cargo de professor titular de epidemiologia, Rio de Janeiro: Escola Nacional de Saúde Pública, Fundação Oswaldo Cruz; 1983.

10. Barbosa FS, Coelho MV. Qualidades de vetor dos hospedeiros de S. mansoni no nordeste do Brasil I: suscetibilidade de A. glabratus e T. centimetralis à infestação por S. mansoni. Publicações Avulsas do Instituto Aggeu Magalhães 1954; 3:55-62.

11. Barbosa FS, Coelho MV, Dobbin JE. Qualidades de vetor dos hospedeiros de S. mansoni no nordeste do Brasil - II: duração da infestação e eliminação de cercarias em A. glabratus. Publicações Avulsas do Instituto Aggeu Magalhães 1954; 3:79-92.

12. Olivier LJ, Barbosa FS, Coelho MV. The influence of infection with Schistosoma mansoni on survival of Austrolorbis glabratus. Publicações Avulsas do Instituto Aggeu Magalhães 1954; 3:63-71.

13. Olivier JL, Barbosa FS. Seasonal studies on Australorbis glabratus (Say) from the localities in Eastern Pernambuco, Brazil. Publicações Avulsas do Instituto Aggeu Magalhães 1955; 4:79-103.

14. Olivier JL, Barbosa FS. Seasonal studies on Tropicorbis centimetralis in Northeastern Brazil. Publicações Avulsas do Instituto Aggeu Magalhães 1955; 4:105-15.

15. Gilles HM, Abdel-Aziz Zaki A, Soussa MH, Samaan SA, Soliman Soliman S, Hassan A, et al. Results of a seven-year snail control project on the endemicity of Schistosoma haematobium in Egypt. Ann Trop Med Parasitol 1973; 67:45-65.
16. World Health Organization. Schistosomisis control. Geneva: World Health Organization; 1985. (WHO Technical Report Series, 728).

17. Pan American Health Organization; WHO Working Group for the Development of Guidance for Identification of American Planorbidae Involved in Schistosomiasis. Document WGAPZ/1 Pan American Health Organization. Washington DC: Pan American Health Organization; 1961.

18. Barbosa FS. Morbidade na esquistossomose [Tese de Doutorado]. Recife: Faculdade de Medicina, Universidade do Recife; 1965.

19. Barbosa FS, Pessoa D, Pinto RF, Barbosa JM, Rodrigues BA. Levantamentos seccionais sobre a esquistossomose no Nordeste do Brasil. I. Estado de Alagoas. Rev Soc Bras Med Trop 1970; 4:129-31.

20. Barbosa FS, Pessoa D, Oliveira O, Barbosa JM, Rodrigues BA. Levantamentos seccionais sobre a esquistossomose no Nordeste do Brasil. II. Estado do Rio Grande do Norte. Rev Soc Bras Med Trop 1970; 4:195-7.

21. Barbosa FS, Pessoa D, Pinto RF, Barbosa JF, Rodrigues BA. Levantamentos seccionais sobre a esquistossomose no Nordeste do Brasil. III. Estado de Pernambuco (Município de S. Lourenço da Mata). Rev Soc Bras Med Trop 1970; 4:269-80.

22. Barbosa FS, Pinto R, Souza O. Control of schistosomiasis in a small northeast Brazilian community. Trans R Soc Trop Med Hyg 1971; 65:206-13. 